\title{
Tumor estromal gástrico apresentando-se com quadro de hemoperitôneo*
}

\author{
Gastric stromal tumor presented as hemorrhagic ascites
}

Francisco Edilson Leite Pinto J unior, ${ }^{1}$ Ariano J osé Freitas de Oliveira, ${ }^{2}$ Álisson Giovani Freitas de Oliveira, ${ }^{3}$ Carlos César Formiga Ramos, ${ }^{4}$ Carlos

César de Oliveira Ramos ${ }^{5}$ e Aldo da Cunha Medeiros ${ }^{6}$

\begin{abstract}
Resumo
0 tumor estromal gástrico (GIST) é uma neoplasia rara que acomete, principalmente, pacientes acima de 50 anos de idade, sem predileção pelo sexo. Entre suas manifestações clínicas, destacam-se anemia, hemorragia gastrointestinal, dor abdominal e massa palpável. 0 caso clínico relatado é de um paciente masculino de 38 anos, apresentando queixas de dor abdominal difusa com predomínio em região epigástrica associada a anemia, perda de peso e constipação intestinal, com dois meses de evolução. Ao exame, encontrava-se com estado geral bom, descorado $(++/ 4+)$, abdome globoso, doloroso à pal pação e sinal de piparote positivo. A endoscopia digestiva alta evidenciou lesão polipóide séssil com ulceração central em grande curvatura, sendo biopsiada e negativa para malignidade. A tomografia computadorizada (TC) de abdome mostrou presença de volumosa lesão ovalar, medindo cerca de $20 \mathrm{x}$ $13 \mathrm{~cm}$ em seus maiores eixos, localizada ao nível do meso e hipogástrio, associada à presença de líquido livre na cavidade abdominal. Submetido à laparotomia exploradora, foi evidenciada ascite hemorrágica importante e presença de grande tumoração cística aderida à grande curvatura do estômago. Realizou-se gastrectomia subtotal com reconstrução em $Y$ de Roux. 0 laudo histopatológico revelou tumor estromal gástrico maligno. Encontra-se assintomático e livre de doença após 24 meses.
\end{abstract}

Palavras-chave: tumor estromal gástrico; neoplasias gástricas; diagnóstico; cirurgia; gastrectomia.

\footnotetext{
* Trabalho apresentado no XXIV Congresso Brasileiro de Cirurgia - 2001.

${ }^{1}$ Ex-residente do IN CA. Professor Assistente do D epartamento de Cirurgia da UFRN. M estre em Cirurgia pela UFPE. Staff da Cirurgia Oncológica da Liga N orte-rio-grandense de Combate ao Câncer. Enviar correspondência para F.E.L.P.J. Rua Pinto M artins 1044 apto.302, Edifício Seychelles, Areia Preta; 59014-060 N atal, RN - Brasil. E-mail: edilsonpinto@uol.com.br

2Professor do Departamento de Cirurgia da UFRN. Aluno do curso de M estrado em Cirurgia da UFRN, N atal, RN - Brasil.

${ }^{3}$ Aluno do curso médico de graduação da UFRN, N atal, RN - Brasil.

${ }^{4}$ Professor do D epartamento de Patologia da UFRN e Chefe do Serviço de patologia da Liga N orterio-grandense de Combate ao Câncer.

${ }^{5}$ M édico Residente de Anatomia Patológica da Unicamp, Campinas, SP - Brasil.

${ }^{6}$ Chefe do D epartamento de Cirurgia e Coordenador da Disciplina de Técnica O peratória e Cirurgia Experimental da UFRN. D outor em

Cirurgia pela UFRJ. Pesquisador do CN Pq. Natal, RN - Brasil.
} 


\begin{abstract}
Gastric stromal tumor is a rare neoplasm, which mainly affects 50 to 70 year-old patients of both genders. Clinical manifestations commonly found are anemia, gastrointestinal hemorrhage, abdominal pain and palpable mass. We report a case of a 38-year-old male patient presenting abdominal pain, especially in epigastrium, associated to anemia, weight loss and intestinal constipation for two months. U pon examination, the patient appeared to be in good conditions, pale skinned, painful globous abdomen, presenting signs of ascites. G astric endoscopy revealed a sessile polypoid fragment with central ulceration in greater curvature. A biopsy of the polyp revealed no malignancy and a computerized tomography (CT) scan of the abdomen demonstrated a large meso to hypogastric mass, measuring $20 \times 13 \mathrm{~cm}$ in large diameters, associated to the presence of ascites. The patient underwent a laparotomy, which showed hemorrhagic ascites and a big cystic tumor in the gastric greater curvature. An extended partial gastrectomy was proceeded. The pathologic diagnosis was a malignant stromal gastric tumor. The patient is now asymptomatic and free of the sickness after 24 months.
\end{abstract}

Key words. gastric stromal tumor; stomach neoplasms; diagnosis; surgery; gastrectomy.

\section{INTRO DUÇÃO}

0 tumor estromal gastrointestinal maligno é uma entidaderara, representando menos de $1 \%$ das neoplasias gástricas, sendo conhecido por seu caráter clínico variável e pela dificuldade em determinar seu comportamento biológico. ${ }^{1,2}$ Cerca de $10 \%$ a $20 \%$ apresentam crescimento exogástrico e geralmente surgem como massa solitária. A maioria dos pacientes são assintomáticos, porém manifestações como dor abdominal, anorexia, náuseas, vômitos e massa abdominal palpável podem estar presentes., $\mathrm{A}$ freqüência de sangramento aumenta nas grandes tumorações com ulceração central. ${ }^{4} \mathrm{C}$ omo esperado em qualquer doença rara, relatos com relação ao diagnóstico, opções terapêuticas e determinantes prognósticos são escassos, com conclusões freqüentemente inconsistentes, limitadas ao pequeno número de casos revisados. 0 objetivo deste trabal ho é apresentar um caso de tumor estromal gástrico cuja manifestação clínica principal foi a presença de hemoperitôneo.

\section{RELATO DO CASO}

Paciente masculino de 38 anos, queixando-se de dor abdominal difusa com predomínio em região epigástrica de forte intensidade, associada a anemia, perda de peso e constipação intestinal, com dois meses de evolução. Ao exame, encontrava-se com estado geral bom, descorado $(+++/ 4+)$, abdome globoso, doloroso à palpação e sinal de piparote positivo.

Foi internado e submetido à endoscopia digestiva alta que evidenciou lesão polipóide séssil com ulceração central em grande curvatura, sendo biopsiada e negativa para malignidade. A tomografia computadorizada (TC) de abdome mostrou presença de volumosa lesão ovalar com características expansivas e contornos regulares bem definidos, caracterizada por formações císticas e septações de permeio, medindo cerca de $20 \times 13 \mathrm{~cm}$ em seus maiores eixos, localizada ao nível do meso e hipogástrio, associada à presença de líquido livre na cavidade abdominal (Figura 1).

0 paciente foi submetido à laparotomia exploradora, sendo evidenciada ascite hemorrágica importante e presença de grande tumoração cística aderida à grande curvatura do estômago (Figuras 2a-c). Realizada a gastrectomia subtotal com reconstrução em $Y$ de Roux, o laudo histopatológico revelou tumor estromal gástrico maligno. Foi realizado o estudo imuno-histoquímico que evidenciou positividade para C-Kit, Vimetina e Ki-67, sendo negativo para Proteína S-100, NES, Desmina, Actina de músculo liso, H H F-35 e CD-34.

0 paciente teve boa evolução no pós-operatório, recebendo alta hospitalar no oitavo dia. $\mathrm{N}$ ão foi indicado tratamento adjuvante. Encontra-se assintomático e livre de doença após 24 meses.

\section{DISCUSSÃO}

0 tumor estromal gastrointestinal é um termo utilizado para as mais comuns neoplasias mesenquimais do trato alimentar, sendo freqüentemente classificadas como tumores da musculatura lisa e schwanomas. C erca de $47 \%$ a $60 \%$ dessas lesões são encontradas no estômago, seguidas de $19 \%$ no intestino delgado, $10 \%$ no cólon e $5 \%$ no esôfago. Os tumores estromais gastrointestinais estão situados na submucosa e musculatura própria do estômago e intestino delgado, geralmente, na grande curvatura gástrica, eo crescimento extramural é raro. ${ }^{5-7}$ 
Figura 1. Volumosa lesão ovalar com contornos regulares bem definidos, medindo cerca de $20 \times 13 \mathrm{~cm}$ em seus maiores eixos.

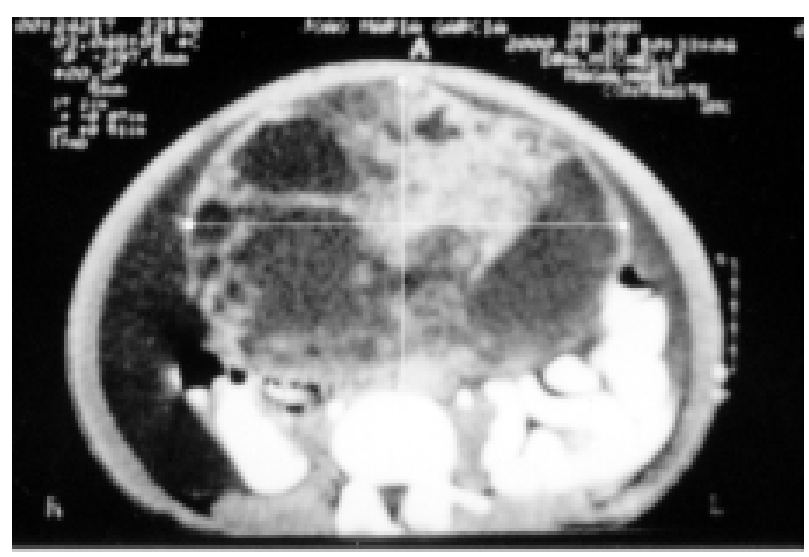

Figura 2a. Grande tumoração cística aderida a grande curvatura do estômago.

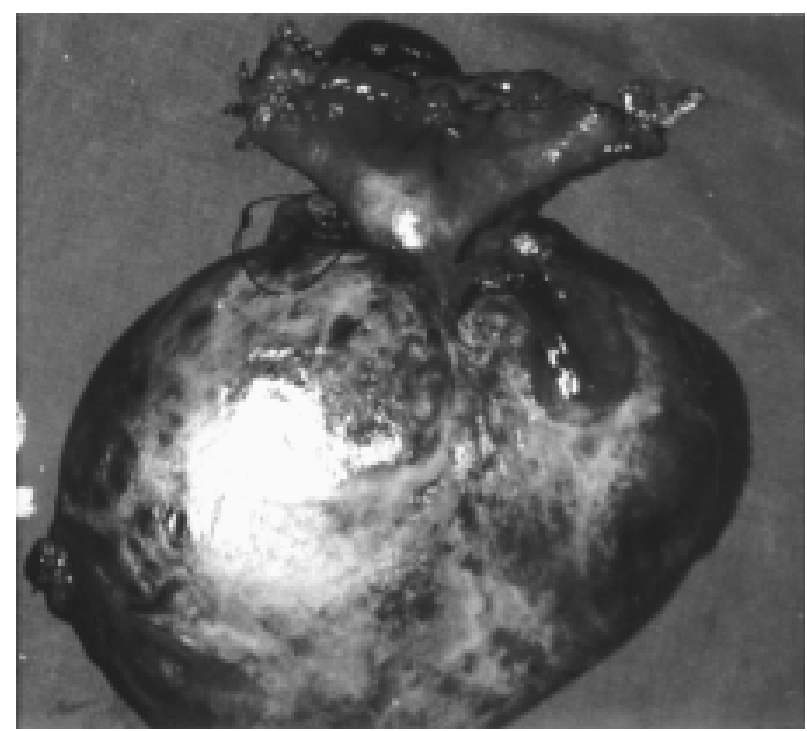

Figura 2b. Lesão polipóide séssil com ulceração central em grande curvatura.

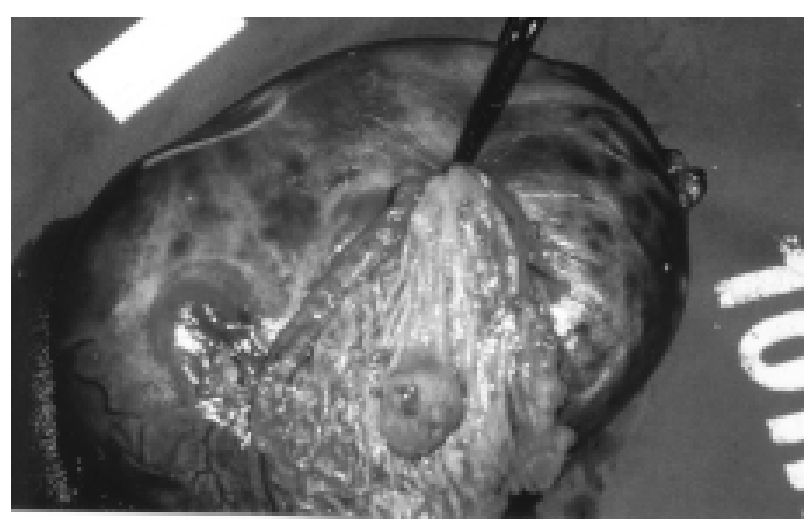

Figura 2c. Tumoração cística com conteúdo hemorrágico no seu interior aderida a grande curvatura do estômago (corte transversal da peça cirúrgica).

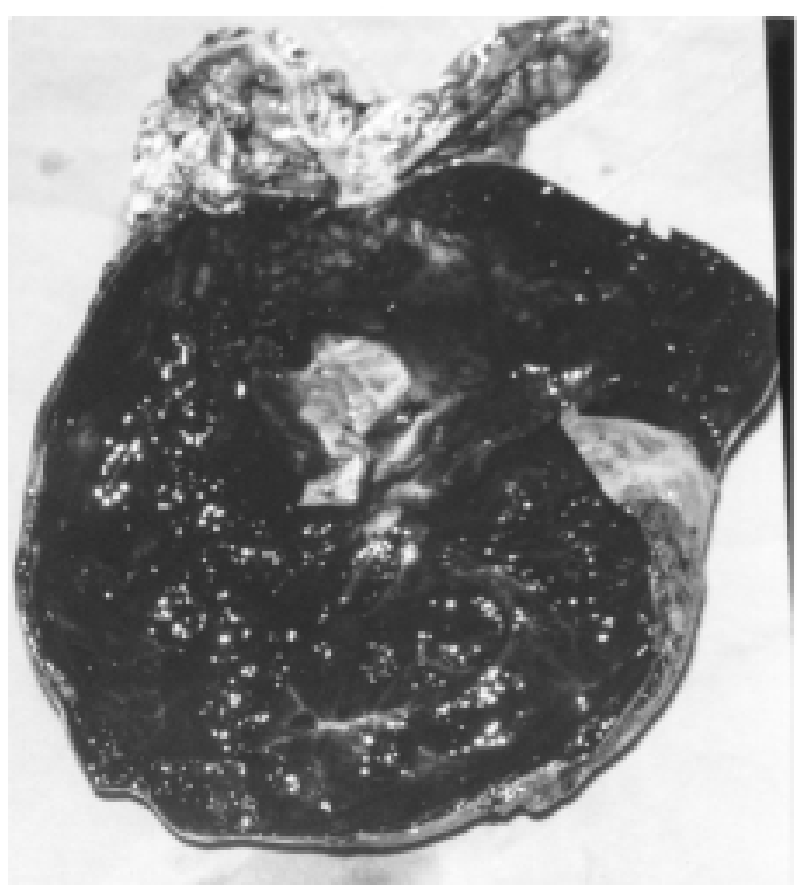

0 tumor estromal gástrico (GIST) é uma neoplasia rara que acomete principalmente pacientes entre 50 e 70 anos de idade, sem predileção pelo sexo e entre cujas manifestações clínicas destacam-se anemia, hemorragia gastrointestinal, dor abdominal emassa pal pável. Sintomas menos comuns, como perda de peso, astenia e saciedade precoce podem ser evidenciados. ${ }^{1,6}$ Todavia, alguns estudos mostram que até $46 \%$ dos pacientes são completamente assintomáticos. A duração desses sintomas varia de três a seis meses antes do diagnóstico. ${ }^{1,3} \mathrm{~A}$ massa tumoral pode ser detectada em menos de $50 \%$ dos pacientes através da endoscopia; dessa forma, freqüentemente há necessidade de estudos radiológicos. A TC é considerada o exame mais específico para o seu diagnóstico quando comparada à endoscopia digestiva alta e seriografia esofagogastroduodenal. ${ }^{7}$

C aracterizam-se microscopicamente por apresentar aspectos histológicos variados. D ois tipos principais de células são observados: células fusiformes e células epitelióides. Os tumores fusocelulares são compostos por fascículos curtos entrelaçados de células com núcleos alongados e citoplasma eosinofílico, fibrilar, podendo, algumas vezes, apresentar padrões sugestivos de diferenciação neural, como estratificação em palisada dos núcleos, crescimento plexiforme e, mais raramente, pigmentação melânica. Tumores epitelióides são formados por ninhos ou cordões de células com núcleos ovóides ou arredondados, com citoplasma abundante e 
eosinofílico, freqüentementecom vacúolos citoplasmáticos perinucleares. Existe certo grau de dificuldade entre os patologistas para o diagnóstico diferencial entre as lesões estromais gastrointestinais benignas e malignas. ${ }^{2,5,7}$

Trupiano et $\mathrm{al}^{8}$ definiram como critérios de malignidade para o GIST, tamanho do tumor maior que $7 \mathrm{~cm}$, celuraridade elevada, invasão da mucosa, alto grau nuclear, contagem de mitoses maior ou igual a 5/50 campos de grande aumento, estroma mixóide, tipo celular misto e ausência de hialinização. D os principais critérios usados nesta diferenciação, o índice mitótico permanece como o mais útil critério histológico nesse diagnóstico, já que em $75 \%$ dos tumores com comportamento biológico agressivo (alto grau de malignidade), há mais do que cinco mitoses por campo

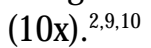

$\mathrm{H}$ á evidências de que esses tumores originem-se das células intersticiais de C arjal. Essas células estão presentes normalmente nos plexos mioentéricos do trato gastrointestinal e funcionam com atividade de marcapasso, regulando a atividade motora do intestino. Assim como as células intersticiais de Cajal, os GIST expressam a proteína KIT, que é o produto do protooncogene $\mathrm{C}-\mathrm{KIT}$, identificada pelo anticorpo monoclonal CD 117. Este anticorpo é observado em $85 \%$ dos casos, sendo sua análise extremamente importante, pois os tumores estromais que expressam c-KIT têm uma alta taxa de resposta ao inibidor da tirosinocinase imatinib, recentemente aprovado pelo FD A norte-americano para tratamento quimioterápico desses tumores. ${ }^{12}$

Com relação à disseminação metastática da doença, Ferrugia et al. ${ }^{9}$ evidenciaram $15 \%$ de comprometimento extragástrico, sendo os sítios mais freqüentemente acometidos, o fígado em $79 \%$ e o pulmão em $15 \%$.

0 tratamento de escolha é a ressecção cirúrgica. Alguns estudos confirmam que a ressecção gástrica em cunha, com margens de 1 a $2 \mathrm{~cm}$, para os tumores menores que $5 \mathrm{~cm}$ de diâmetro seja considerada a cirurgia adequada. ${ }^{10,11}$ Como 0 envolvimento linfonodal no GIST é raro, não há necessidade de realizar a linfadenectomia padrão como indicado nos casos de adenocarcinomas gástricos. ${ }^{10} \mathrm{~N}$ a presença de doença metastática, a lesão primária deve ser ressecada de forma paliativa, porquea sobrevida pode ser longa mesmo com a disseminação tumoral.

0 prognóstico desses tumores depende do grau histopatológico, tamanho do tumor e evidência de metástases. A sobrevida em cinco anos está presente em $90 \%$ dos casos para os tumores de baixo grau e cai para $50 \%$ nos de alto grau de malignidade. . $^{9-11}$

$\mathrm{N}$ o caso ora relatado a inusitada manifestação clínica inicial, através do hemoperitôneo, parece não ter afetado a sobrevida do paciente, já que ele encontra-se assintomático e livre de doença após 24 meses da cirurgia.

\section{REFERÊNCIAS BIBLIO G RÁFICAS}

1. Shimoji $H, M$ iyazato $H, N$ akachi $A$, Shiraishi M, M uto Y. Pedunculated exogastric leiomyosarcoma: case report and brief literaturereview. Clin Imaging 1999;23:94-8.

2. Ruiz AR Jr, N assar A J, Fromm H . M ultiplemalignant gastric stromal tumors presenting with $\mathrm{GI}$ bleeding: a case report a rewiew of the literature. Gastrointest Endosc 2000;51:225-8.

3. Burchett KD , C arungi RS. G astric leiomyosarcoma presenting as a sentinel hemorrhage. J Am 0 steopath Assoc 1999;99:533-6.

4. Bagnolo F, Bonassi U, Scelsi R, Testoni PA. Gastric stromal tumor: a rare neoplasm presenting with gastointestinal bleeding. Eur J Gastroenterol 1998;10:791-4.

5. Barrier A, H uguier $M$, Levard $H$, M ontariol T, Fagniez PL, Sauvanet $A$. Tumeursgastriques conjonctives. Resultatsd'une étudemulticentrique. Chirurgic 1999;124:494-502.

6. Akwari OE, D ozois RR, Weiland LH, Beahrs OH . Leiomyosarcoma of the small and large bowel. Cancer 1978;42:1375-84.

7. Sheen-Chen SM, Chou FF, Eng H L, Chen JJ, LeeCM, Wan YL. Gastric leiomyosarcoma: a clinicopathological review. Eur J Surg 1994;160:681-7.

8. TrupianoJK, Stewart RE, M isick C, Appelman H D. Gastric stromal tumors: a clinicopathologic study of 77 cases with correlation of features with nonaggressiveand aggressiveclinical behaviors. Am J Surg Pathol 2002;26(6):705-14.

9. Ferrugia $\mathrm{G}, \mathrm{Kim} \mathrm{CH}, \mathrm{G}$ rant CS, Zinsmeister AR . Leiomyosarcoma of the stomach: determinants of long-term survival. M ayo Clin Proc 1992;67:533-6.

10. Katai H , Sasako M , Sano T, M aruyamaK. Wedgeresection of the stomach for gastric leiomysarcoma. $\mathrm{Br}$ J Surg 1997;84:560-1.

11. G rant CS, G oellner JR. G astric leiomyosarcoma: prognostic factors and surgical management. Arch Surg 1991;126:985-9.

12. Buzaid AC, D zik C. Sarcomas departes moles. In: Buzaid $A C, C$ utait $R$, editores. M anual de oncologia clínica do H ospital Sírio Libanês. 1a ed. Rio deJ aneiro: Reichmann \& Affonso; 2002. p. 261-8. 